\title{
BIFURCATION OF HOMOCLINICS
}

\author{
JACOBO PEJSACHOWICZ
}

(Communicated by Carmen C. Chicone)

\begin{abstract}
We show that homoclinic trajectories of nonautonomous vector fields parametrized by a circle bifurcate from the stationary solution when the asymptotic stable bundles of the linearization at plus and minus infinity are "twisted" in different ways.
\end{abstract}

\section{INTRODUCTION}

The purpose of this paper is to explain the appearance of homoclinic solutions of nonautonomous differential equations in terms of the asymptotic behavior of their linearization. The functional analytic methods used in proofs of existence of homoclinic trajectories of differential equations are mainly of three types. The first uses Melnikov functions in order to prove the persistence of homoclinic orbits under a small change of parameter. The second, typical of Hamiltonian systems, reduces the problem of existence of a homoclinic orbit to the one of existence of a nontrivial critical point of the action functional and then applies various generalizations of the mountain pass theorem. Here we will use the third approach which parallels the analysis of Hopf-bifurcation of periodic orbits from an equilibrium. Instead of focusing on the existence of a single homoclinic, we will consider a family of differential equations parametrized by a circle. Further, using a general bifurcation principle for Fredholm maps, we will show that a branch of homoclinics bifurcating from the stationary solution appears whenever the asymptotic stable bundles of the linearization at plus and minus infinity are twisted differently, i.e., nonisomorphic. The study of homoclinics based on bifurcation theory is far from being new [13]. What is new here is that the appearance of homoclinics is a consequence of the nontrivial topology of the circle. It has been observed elsewhere that the topology of the parameter space produces interesting and, sometimes, unexpected global effects on dynamics. For example, it is accountable for the appearance of Berry's phase in the adiabatic approximation of linear Hamiltonian systems when the Hamiltonian moves around a closed loop in the parameter space [3]. In [9] the authors construct a refined version of the Conley index associated to a family of flows parametrized by a circle, which encodes information about invariant sets of the flows that cannot be obtained from a local analysis. Our arguments here will be of the same type. We first translate the problem into one of bifurcation of zeroes of a family of Fredholm maps. Then we will consider the index bundle of the family

Received by the editors August 3, 2006.

2000 Mathematics Subject Classification. Primary 34C23, 58E07; Secondary 37G20, 47A53.

Key words and phrases. Differential equations, homoclinics, bifurcation, index bundle. 
of linearizations at points of the trivial branch given by the stationary solutions of the equation. The index bundle of a family of Fredholm operators is a refinement of the ordinary index of a Fredholm operator which takes into account the topology of the parameter space. It is known that nonorientability of the index bundle entails bifurcation of zeroes of Fredholm maps [6]. On the other hand an elementary index theorem, Proposition 3.1, computes the index bundle in terms of the asymptotic stable bundles at plus and minus infinity, relating the appearance of homoclinics to the asymptotic behavior of coefficients of linearized equations. The precise result is stated in Theorem 2.1 of Section 2. Section 3 is devoted to the proof of this theorem. Section 4 is an example.

\section{THE MAIN RESULT}

We consider the system

$$
\left\{\begin{array}{l}
u^{\prime}(t)-g(\lambda, t, u(t))=0 \\
\lim _{t \rightarrow+\infty} u(t)=0=\lim _{t \rightarrow-\infty} u(t),
\end{array}\right.
$$

depending on a parameter $\lambda$ belonging to the circle $S^{1}$.

Here $g: S^{1} \times \mathrm{R} \times \mathrm{R}^{n} \rightarrow \mathrm{R}^{n}$ is smooth, both $g$ and $D_{u} g$ are bounded and moreover $g(\lambda, t, 0)=0$.

Because of the last assumption, for every $\lambda \in S^{1}$, the pair $(\lambda, 0)$ is a trivial solution of (2.1). We are interested in nontrivial solutions, i.e., solutions $(\lambda, u)$ with $u \neq 0$.

For each, $\lambda \in S^{1}$, the linearization of (2.1) at $u=0$ is given by

$$
\left\{\begin{array}{l}
u^{\prime}(t)-A(\lambda, t) u(t)=0 \\
\lim _{t \rightarrow+\infty} u(t)=0=\lim _{t \rightarrow-\infty} u(t),
\end{array}\right.
$$

where $A(\lambda, t)=D_{u} g(\lambda, t, 0)$.

We will assume:

(A1) As $t \rightarrow \pm \infty$ the family $A(\lambda, t)$ converges uniformly to a family of matrices $A(\lambda, \pm \infty)$, such that $A(\lambda, \pm \infty)$ has no eigenvalues on the imaginary axis.

(A2) For some fixed $\lambda_{0} \in S^{1}$ both (2.2) and its adjoint problem admit only the trivial solution $u \equiv 0$.

As a consequence of $(\mathrm{A} 1)$, the map $\lambda \rightarrow A(\lambda, \pm \infty)$ is continuous. By standard perturbation theory the spectral projectors corresponding to the spectrum of $A(\lambda, \pm \infty)$ on the left and right half plane are continuous as well. Hence the families of vector spaces $E^{s}(\lambda, \pm \infty)$ and $E^{u}(\lambda, \pm \infty)$, whose elements are the generalized real eigenvectors of $A(\lambda, \pm \infty)$ corresponding to the eigenvalues with negative and positive real parts, define a pair of vector bundles $E^{s}( \pm \infty)$ and $E^{u}( \pm \infty)$ over $S^{1}$. This pair decomposes the trivial bundle $\Theta\left(\mathrm{R}^{n}\right)=S^{1} \times \mathrm{R}^{n}$ into a direct sum:

$$
E^{s}( \pm \infty) \oplus E^{u}( \pm \infty)=\Theta\left(\mathrm{R}^{n}\right) .
$$

In what follows $E^{s}( \pm \infty)$ and $E^{u}( \pm \infty)$ will be called stable and unstable asymptotic bundles at $\pm \infty$ since they can be characterized in terms of the decay of solutions of the linear system associated to $A(\lambda, \pm \infty)$.

Together with (A1), condition (A2) imposes some extra restrictions on the coefficients of (2.2). Indeed, if $W_{\lambda}^{s / u}=\left\{u(0) / u^{\prime}(t)=A(\lambda, t) u(t), \lim _{t \rightarrow+\infty /-\infty} u(t)=0\right\}$ are the stable and unstable subspaces of the system $u^{\prime}(t)=A(\lambda, t) u(t)[10]$, then solutions of (2.2) are in a one-to-one correspondence with elements of $W_{\lambda}^{s} \cap W_{\lambda}^{u}$. 
The stable and unstable subspaces for the adjoint equation are the orthogonals of $W_{\lambda}^{s / u}$. Hence, assumption (A2) is equivalent to

$$
W_{\lambda_{0}}^{s} \cap W_{\lambda_{0}}^{u}=\{0\} \text { and } W_{\lambda_{0}}^{s}+W_{\lambda_{0}}^{u}=\mathrm{R}^{n} .
$$

It is well known that, if (A1) holds, then the image of $W_{\lambda}^{s / u}$ under the flow associated to the above equation converges, as $t \rightarrow \pm \infty$, to $E^{s / u}(\lambda, \pm \infty)$. In particular, it follows from (2.3) that for $\lambda=\lambda_{0}$ and hence for all $\lambda \in S^{1}$,

$$
\operatorname{dim} E^{s}(\lambda,-\infty)=\operatorname{dim} E^{s}(\lambda,+\infty) .
$$

The equality (2.5) restricts the behavior of $A(\lambda, t)$ at $\pm \infty$. Notice, however, that (2.5) is always verified in the Hamiltonian case, because the stable and unstable subspaces of a Hamiltonian system are Lagrangian.

Our main theorem relates the appearance of homoclinic solutions to the topology of the asymptotic stable bundles $E^{s}( \pm \infty)$.

Each vector bundle $E$ over $S^{1}$ comes with an associated topological invariant $w_{1}(E) \in \mathrm{Z}_{2}$ which measures whether the bundle is "twisted" or "untwisted". In order to define it, let us consider $p:[0,2 \pi] \rightarrow S^{1}$ defined by $p(\theta)=e^{i \theta}$ and let us choose a frame for the pull-back $p^{*} E$, i.e., a basis $\left\{e_{1}(\theta), \ldots, e_{k}(\theta)\right\}$ of $E_{p(\theta)}$ continuously depending on $\theta$. Such a frame always exists. We define $w_{1}(E) \in \mathrm{Z}_{2}$ by

$$
(-1)^{w_{1}(E)}=\operatorname{sign} \operatorname{det} C,
$$

where $C$ is the matrix expressing the basis $\left\{e_{i}(2 \pi), 1 \leq i \leq k\right\}$ in terms of the basis $\left\{e_{i}(0), 1 \leq i \leq k\right\}$.

It is easy to see that $w_{1}(E)$ is independent from the choice of the frame. Clearly, $w_{1}(E)=0$ if and only if $E$ is orientable, i.e., it admits a frame with $\operatorname{det} C>0$. Thus, via the isomorphism $\mathbf{H}^{1}\left(S^{1} ; \mathrm{Z}_{2}\right) \cong \mathrm{Z}_{2}, w_{1}(E)$ can be identified with the first Stiefel-Whitney class of $E$.

A vector bundle over $S^{1}$ is orientable if and only if it is trivial, i.e., isomorphic to the product bundle $\Theta\left(\mathrm{R}^{k}\right)$. Indeed if $\operatorname{det} C>0$, there is a path $C(\theta)$ with $C(0)=C$ and $C(2 \pi)=\mathrm{Id}$. Then $f_{i}(\theta)=C(\theta) e_{i}(\theta)$ is a frame such that $f_{i}(0)=f_{i}(2 \pi)$ and hence $\Phi\left(e^{i \theta}, x_{1}, \ldots, x_{k}\right)=\left(e^{i \theta}, \sum x_{i} f_{i}(\theta)\right)$ is an isomorphism between $\Theta\left(\mathrm{R}^{k}\right)$ and $E$. The converse is clear. Therefore, $w_{1}(E)$ is either zero or one depending on whether the vector bundle $E$ is isomorphic to a product bundle.

The space $H^{1}\left(\mathrm{R} ; \mathrm{R}^{n}\right)$ of all absolutely continuous functions $u \in L^{2}\left(\mathrm{R} ; \mathrm{R}^{n}\right)$ with square integrable derivative is a natural function space for our problem since any function $u \in H^{1}\left(\mathrm{R} ; \mathrm{R}^{n}\right)$ is continuous and has the property that $\lim _{t \rightarrow \pm \infty} u(t)=0$. By bootstrap, any $H^{1}$-solution of (2.1) is $C^{1}$ and decays to 0 at $\pm \infty$ together with its derivative.

Theorem 2.1. If the system (2.1) verifies (A1), (A2) and if

$$
w_{1}\left(E^{s}(+\infty)\right) \neq w_{1}\left(E^{s}(-\infty)\right),
$$

then for all $\epsilon$ small enough there is a solution $(\lambda, u)$ of $(2.1)$ with $\|u\|_{H^{1}}=\epsilon$.

Remark 2.2. A point $\lambda_{*} \in S^{1}$ is a bifurcation point for homoclinic solutions of (2.1) from the stationary solution $u=0$, if there is sequence $\left(\lambda_{n}, u_{n}\right) ; \lambda_{n} \in S^{1}$, $u_{n} \neq 0$ solution of $(2.1)$ with $\lambda_{n} \rightarrow \lambda_{*}$ and $u_{n} \rightarrow 0$ in $H^{1}$. By compactness of $S^{1}$ it follows that, under the hypothesis of the above theorem, there must be at least one bifurcation point for homoclinic trajectories $\lambda \in S^{1}$. However the theorem 
does not provide the exact location of bifurcation points. On the other hand, in contradistinction to the local bifurcation invariants, (2.7) holds for any asymptotically vanishing smooth nonlinear perturbation of (2.1) and, more importantly, is computable directly from the coefficients of the linearization.

\section{Proof of Theorem 2.1}

Let $X=H^{1}\left(\mathrm{R} ; \mathrm{R}^{n}\right), Y=L^{2}\left(\mathrm{R} ; \mathrm{R}^{n}\right)$ and let $f: S^{1} \times X \rightarrow Y$ be defined by

$$
f(\lambda, u)(t)=u^{\prime}(t)-g(\lambda, t, u(t)) .
$$

Using the embedding of $X$ into $C^{0}(\mathrm{R})$, it is easy to show that $f$ is $C^{1}$ and the Frechet derivative $D_{u} f(\lambda, 0)$ is the operator $L_{\lambda}: X \rightarrow Y$ defined by

$$
\left[L_{\lambda} u\right](t)=u^{\prime}(t)-A(\lambda, t) u(t) .
$$

We will show that the restriction of $f$ to a neighborhood of the set of trivial solutions is a family of $C^{1}$-Fredholm maps of index 0 . With this, our result will be a consequence of the homotopy principle of the degree constructed in [11] for this class of maps and the result in [6] relating the parity of a closed path of Fredholm operators with the first Steifel-Whitney class of its index bundle.

Below we recall the properties of the degree and of the index bundle that will be used in the proof.

The construction of the degree in [11] is based on the notion of parity of a path of Fredholm operators of index 0 . Given a path $L:[a, b] \rightarrow \Phi_{0}(X, Y)$ with invertible end points and transverse to the stratified one-codimensional variety $\Sigma$ of all noninvertible Fredholm operators, its parity $\sigma(L) \in \mathrm{Z}_{2}$ is defined as the number of intersection points of the path with the top stratum $\Sigma_{1}=\{T \in \Sigma \mid \operatorname{dim} \operatorname{ker} T=1\}$ counted mod 2. This definition extends to general paths with invertible end points either by approximation with transversal paths as in [7] or by using parametrices as in $[6]$.

Let $\mathcal{O} \subset X$ be an open simply connected set and let $f: \mathcal{O} \rightarrow Y$ be a $C^{1}$-Fredholm map of index 0 that is proper on closed bounded subsets of the domain. Using parity, we can assign to each regular point of the map $f$ an orientation $\epsilon(x)= \pm 1$ with the same properties as the sign of the Jacobian determinant in finite dimensions. For this we choose a fixed regular point $b$ of $f$ (called base point) and then the corresponding orientation $\epsilon_{b}(x)$ at any regular point $x$ is uniquely defined by the requirement $\epsilon_{b}(x)=(-1)^{\sigma(D f \circ \gamma)}$, where $\gamma$ is any path in $\mathcal{O}$ joining $b$ to $x$. Since $\mathcal{O}$ is simply connected, the independence from the choice of the path follows from the homotopy invariance of the parity. Let $\Omega$ be an open bounded set with closure contained in $\mathcal{O}$ such that $0 \notin f(\partial \Omega)$ and 0 is a regular value of the restriction of $f$ to $\Omega$, then the base point degree of $f$ in $\Omega$ is defined by

$$
\operatorname{deg}_{b}(f, \Omega, 0)=\sum_{x \in f^{-1}(0)} \epsilon_{b}(x) .
$$

It was proved in [11] that this assignment extends to an integral-valued degree theory for $C^{1}$-Fredholm maps defined on simply connected sets, which are proper on closed bounded subsets of its domain. The base point degree is invariant under homotopies only up to sign and, as a matter of fact, no degree theory for general Fredholm maps can be homotopy invariant. However, the change in sign along a homotopy can be determined as follows [11, Theorem 5.1]: Let $h: I \times \mathcal{O} \rightarrow Y$ be a 
homotopy and let $\Omega$ be an open bounded subset of $X$ such that $0 \notin h([0,1] \times \partial \Omega)$. Assume (for simplicity) that $b$ is a regular point both of $h_{0}$ and $h_{1}$, then

$$
\operatorname{deg}_{b}\left(h_{0}, \Omega, 0\right)=(-1)^{\sigma(H)} \operatorname{deg}_{b}\left(h_{1}, \Omega, 0\right),
$$

where $H$ is the path $t \rightsquigarrow D h_{t}(b)$.

Next let us recall the properties of the index bundle. The Atiyah-Janich construction [2] associates to each family $L: \Lambda \rightarrow \Phi(X, Y)$ of Fredholm operators parametrized by a compact space $\Lambda$ an element Ind $L$ of the Grothendieck group $K O(\Lambda)$. This latter is, by definition, the group completion of the abelian semigroup $\operatorname{Vect}^{()}(\Lambda)$ of all isomorphisms classes of vector bundles over $\Lambda$. Namely, it is the quotient of the semigroup $\operatorname{Vect}^{()}(\Lambda) \times \operatorname{Vect}^{(}(\Lambda)$ by the diagonal sub-semigroup. The elements of $K O(\Lambda)$ are called virtual bundles. Each virtual bundle can be written as a difference $[E]-[F]$ where $E, F$ are vector bundles over $\Lambda$ and $[E]$ denotes the equivalence class of $(E, 0)$. Moreover we have that $[E]-[F]=0$ in $K O(\Lambda)$ if and only if the vector bundles $E$ and $F$ are stable equivalent, i.e., they become isomorphic after the addition of a trivial bundle to both sides.

The following is a variant of the Atiyah-Janich construction [6]: using the compactness of $\Lambda$ one can find a finite-dimensional subspace $V$ of $Y$ such that

$$
\operatorname{Im} L_{\lambda}+V=Y \text { for any } \lambda \in \Lambda .
$$

It follows from (3.5) that the family of finite-dimensional spaces $E_{\lambda}=L_{\lambda}^{-1}(V)$ forms a vector bundle $E$ over $\Lambda$. By definition, the index bundle or analytical index of the family is the virtual bundle Ind $L=[E]-[\Theta(V)]$, where $\Theta(V)=\Lambda \times V$ denotes the trivial vector bundle with fiber $V$. If $V \subset W$ are two subspaces verifying the transversality condition (3.5) and $E \subset F$ are the corresponding vector bundles, the restriction of the family $L$ to $F$ induces an isomorphism from $F / E$ onto the trivial bundle with fiber $W / V$. Since exact sequences of vector bundles split, it follows that $F$ is isomorphic to a direct sum of $E$ with $\Theta(W / V)$, and from this it follows that $[E]-[\Theta(V)]=[F]-[\Theta(W)]$ in $K O(\Lambda)$. Thus Ind $L$ is well defined. It is additive under direct sums and moreover Ind $L=0$ if $L$ is a family of isomorphisms. From the functoriality of this construction it follows easily that Ind $L$ is invariant under homotopies of Fredholm families and, in particular, the index bundle is invariant under perturbations by a family of compact operators. We will need also the logarithmic property of the index bundle. Namely, if $L: \Lambda \rightarrow \Phi(X, Y)$ and $M$ : $\Lambda \rightarrow \Phi(Y, Z)$ are two families, then Ind $(L \circ M)=$ Ind $L+$ Ind $M$. For a simple proof it is enough to notice that in the construction of the index bundle, one can take any finite-dimensional subbundle of $\Lambda \times Y$ transverse to $L$ in the sense of (3.5), then the above formula follows immediately from the fact that $E=L^{-1} \Theta(V)$ is transverse to the family $M$.

Using the above properties, we compute the index bundle of the family $L$ defined by (3.2) in terms of the asymptotic stable bundles $E^{s}( \pm \infty)$.

Proposition 3.1. The family $L: S^{1} \rightarrow \mathcal{L}(X ; Y)$ defined by (3.2) verifies that

i) $L_{\lambda}$ is Fredholm of index 0 for all $\lambda \in S^{1}$;

ii) Ind $L=\left[E^{s}(+\infty)\right]-\left[E^{s}(-\infty)\right] \in K O\left(S^{1}\right)$.

Proof. We split $\mathrm{R}$ into $\mathrm{R}=\mathrm{R}^{+} \cup R^{-}$where $R^{ \pm}=[0, \pm \infty)$ and we denote with $X^{ \pm}, Y^{ \pm}$the spaces $H^{1}\left(R^{ \pm} ; \mathrm{R}^{n}\right)$ and $L^{2}\left(\mathrm{R}^{ \pm} ; \mathrm{R}^{n}\right)$, respectively. Let us consider the operators $L_{\lambda}^{ \pm}: X^{ \pm} \rightarrow Y^{ \pm}$defined as in (3.2) using the restrictions of $A(\lambda,$.$) to \mathrm{R}^{ \pm}$. 
We will show that $L_{\lambda}^{ \pm}$are Fredholm and compute the index bundles of $L^{ \pm}$. First we notice that if $M_{\lambda}^{ \pm}: X^{ \pm} \rightarrow Y^{ \pm}$is defined by

$$
\left[M_{\lambda}^{ \pm} u\right](t)=u^{\prime}(t)-A(\lambda, \pm \infty) u(t)
$$

then $K_{\lambda}^{ \pm}=M_{\lambda}^{ \pm}-L_{\lambda}^{ \pm}$is a compact operator for each $\lambda \in S^{1}$.

To see this, let us take for each $m \in \mathrm{N}$ a smooth function $\phi_{m}$ defined on $\mathrm{R}_{+}$ such that $\phi_{m} \equiv 1$ on $[0, m-1]$ and $\phi_{m} \equiv 0$ on $[m,+\infty)$. Then, $K_{\lambda}^{+}=\lim _{m \rightarrow \infty} K_{\lambda}^{m}$ where

$$
\left[K_{\lambda}^{m} u\right](t)=\phi_{m}(t)[A(\lambda,+\infty)-A(\lambda, t)] u(t) .
$$

But $K_{\lambda}^{m}$ is a compact operator for every $m$ because it factorizes through the inclusion $H^{1}\left([0, m] \mathrm{R}^{n}\right) \subset L^{2}\left(\mathrm{R}^{+} ; \mathrm{R}^{n}\right)$, which is compact. This shows that $L$ is a compact perturbation of $M$. On the other hand it is well known that $M_{\lambda}$ are surjective with $\operatorname{ker} M_{\lambda}=E^{s}(\lambda,+\infty)$. The second assertion is clear. For the first it is enough to observe that a right inverse for $M_{\lambda}$ is given by

$$
S_{\lambda}(v)(t)=\int_{0}^{t} P_{\lambda} e^{(s-t) A_{\lambda}(s)} v(s) d s+\int_{t}^{\infty}\left(\mathrm{id}-P_{\lambda}\right) e^{(t-s) A_{\lambda}(s)} v(s) d s,
$$

where $P_{\lambda}$ is the projector onto $E^{s}(\lambda,+\infty)$. Thus $M_{\lambda}^{+}$and hence also $L_{\lambda}^{+}$are Fredholm. Moreover by invariance of the index bundle by compact perturbations Ind $L^{+}=$Ind $M^{+}=\left[E^{s}(+\infty)\right]$ and similarly Ind $L^{-}=\left[E^{u}(-\infty)\right]$.

In order to compute the index of $L$ let us notice first of all that the restriction map $I: Y \rightarrow Y^{-} \oplus Y^{+}$defined by $I v=\left(v_{\mid \mathrm{R}^{-}}, v_{\mid \mathrm{R}^{+}}\right)$is an isomorphism, while the restriction map $J: X \rightarrow X^{-} \oplus X^{+}$is a monomorphism whose image is given by $\operatorname{Im} J=\left\{\left(u^{-}, u^{+}\right) / u^{-}(0)=u^{+}(0)\right\}$. Hence, if $\psi\left(u^{-}, u^{+}\right)=u^{-}(0)-u^{+}(0)$, then $\operatorname{Im} J=\operatorname{ker} \psi$ and therefore $J$ is Fredholm of index $-n$. From the commutative diagram

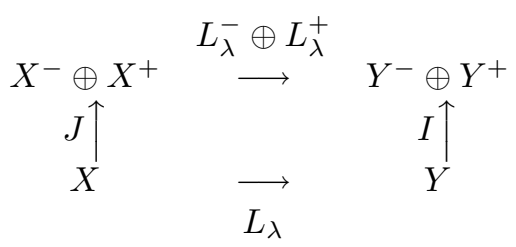

it follows that $L_{\lambda}$ is Fredholm. Moreover by continuity of the index and assumption (A2), the index of $L_{\lambda}$ must be 0 . This proves i). Now ii) follows from (3.9) using the logarithmic property of the index bundle. Indeed considering $I$ and $J$ as constant families of Fredholm operators Ind $I=0$, Ind $J=-\left[\Theta\left(\mathrm{R}^{n}\right)\right]$. But then by $(2.3)$

$$
\text { Ind } L=\left[E^{u}(-\infty)\right]+\left[E^{s}(+\infty)\right]-\left[\Theta\left(\mathrm{R}^{n}\right)\right]=\left[E^{s}(+\infty)\right]-\left[E^{s}(-\infty)\right] \text {. }
$$

With this established, let us come back to the proof of Theorem 2.1. Since $L$ takes values in the open set $\Phi_{0}(X ; Y)$, it follows that, for $\delta$ small enough, the restriction of $f$ to $S^{1} \times B(0, \delta)$ is a $C^{1}$ family of Fredholm maps of index 0 . Since Fredholm maps are locally proper, it will be also a proper map taking $\delta$ small enough. For definiteness, let $\lambda_{0}=1 \in S^{1}$. Since 0 is a regular point of $f_{1}$, by the inverse function theorem we can suppose that, for $\delta$ small enough, 0 is the only solution of $f_{1}(x)=0$ in $B(0, \delta)$. If for some $\epsilon<\delta$ there are no solutions of $f(\lambda, u)=0$ with $\|u\|=\epsilon$, then the map $h:[0,1] \times \overline{B(0, \epsilon)} \rightarrow Y$ defined by $h(\theta, u)=f(\exp i \theta / 2 \pi, u)$ is an admissible homotopy. On the other hand we can take $b=0$ as the base point for both $h_{0}=h_{1}$ and then by formula $(3.3) \operatorname{deg}_{b}\left(h_{0}, B(0 . \epsilon), 0\right)=\operatorname{deg}_{b}\left(h_{1}, B(0 . \epsilon), 0\right)=1$. 
By the homotopy formula (3.4) the parity of the closed path $\theta \rightsquigarrow L_{\exp i \theta / 2 \pi}$ must be 0 . On the other hand, it was proved in [6, Proposition 2.7] that the parity of a closed path coincides with the first Stiefel-Whitney class of its index bundle. Thus, we get $0=w_{1}($ Ind $L)=w_{1}\left(E^{s}(+\infty)\right)-w_{1}\left(E^{s}(-\infty)\right)$, which contradicts the hypothesis.

Remark 3.2. What is crucial to the proof of Proposition 3.1 is that an asymptotically hyperbolic matrix function has an exponential dichotomy [4] on both half lines $\mathrm{R}^{ \pm}$. Indeed, a version of Proposition 3.1 ii) holds under this sole assumption if the corresponding projectors $P_{\lambda}^{+}, P_{\lambda}^{-}$depend continuously on the parameter. In this case, formula (3.8) involving the fundamental matrix of the system defines a right inverse for $L^{+}$and similarly for $L^{-}$. Now, diagram (3.9) gives Ind $L=\left[\operatorname{Im} P^{+}\right]-\left[\operatorname{Im} P^{-}\right]$. Similar arguments can be used in other function spaces as well, extending to families of operators the index computations in $[12,10]$. Fredholm properties of differential operators on infinite-dimensional spaces have been recently established in $[1,8]$. An extension of Proposition 3.1 to this case would open the possibility of applying the general principle described here not only to bifurcation of various types of bounded solutions of ordinary differential equations but also to partial differential equations on unbounded domains, for example of the type considered in [8].

\section{An eXAmple}

Let $a(t)$ be any smooth function which vanish identically in a neighborhood of 0 , such that $a(t) \equiv-1$ outside of a compact subset of the real line. For $\lambda=e^{i \theta}, 0 \leq$ $\theta<2 \pi$, let

$$
A(\lambda, t)=\left\{\begin{array}{ll}
a(t) R_{\theta} & \text { if } t \geq 0, \\
a(t) R_{\pi} & \text { if } t \leq 0,
\end{array} \quad \text { where } R_{\theta}=\left(\begin{array}{rr}
\cos \theta & \sin \theta \\
\sin \theta & -\cos \theta
\end{array}\right)\right.
$$

is the symmetry with respect to the line $l_{\lambda}$ generated by $(\cos \theta / 2, \sin \theta / 2)$.

Here $E^{s}(+\infty)=\left\{(\lambda, u) \in S^{1} \times \mathrm{R}^{2} / u \in l_{\lambda}\right\}$ is the tautological line bundle over the real projective space $\mathrm{R} P^{1} \sim S^{1}$, i.e., the Moebius strip, with $C=-\mathrm{id}$. $E^{u}(+\infty)=E^{s}(+\infty)^{\perp}$ while $E^{s / u}(-\infty)$ are the trivial bundles $\Theta\left(l_{-1}\right)$ and $\Theta\left(l_{1}\right)$, respectively. Moreover, due to the special form of $A(\lambda, t), E_{\lambda}^{s}(+\infty)$ is positively invariant under the flow of $u^{\prime}=A(\lambda, t) u$ and $E^{u}(-\infty)$ is negatively invariant. It follows from this that $W_{\lambda}^{s}=E_{\lambda}^{s}(+\infty)=l_{\lambda}$ and $W_{\lambda}^{u}=E_{\lambda}^{u}(-\infty)=l_{1}$. Therefore $W_{\lambda}^{s}$ and $W_{\lambda}^{u}$ have a nontrivial intersection only when $\lambda=1$. By Proposition 3.1 i) $L_{\lambda}$ is an isomorphism for all $\lambda \neq 1$. From the implicit function theorem it follows that $\lambda=1$ is the only possible bifurcation point of homoclinic solutions for any nonlinear problem of the form $u^{\prime}(t)-A(\lambda, t) u(t)+g(\lambda, t, u(t))=0$ with $g(\lambda, t, u)=o(\|u\|)$. In fact, either using the degree theory described above or simply by applying the Crandall-Rabinowitz bifurcation theorem [5] it can be shown that if $g=o(\|u\|)$, then a branch of homoclinics bifurcates from the stationary solution at $\lambda=1$. On the other hand, since $\omega_{1}\left(E^{s}(-\infty)\right)=1 \neq \omega_{1}\left(E^{s}(+\infty)\right)=0$, the hypotheses of Theorem 2.1 are verified by any smooth perturbation $g$ with $g(\lambda, t, 0)=0$, $\lim _{t \rightarrow \pm \infty} g(\lambda, t, u)=0$ and such that, for some $\lambda_{0} \neq 1, g_{\lambda_{0}}$ is small enough in the $C^{1}$ norm. Therefore, equation $u^{\prime}(t)-A(\lambda, t) u(t)+g(\lambda, t, u(t))=0$ will have homoclinic solutions arbitrarily close to 0 for any $g$ as above. 
In order to show that we cannot dispense with (A2), let us consider a similar problem in $R^{3}$ by taking the previous $2 \times 2$ system together with a third uncoupled equation $u_{3}^{\prime}(t)-\arctan t u_{3}(t)=0$. This only has the effect of adding a trivial line bundle to the previous $E^{s}(-\infty)$ and hence the invariant $\omega_{1}$ remains unchanged. But now the adjoint equation to (2.2) has a homoclinic solution $u^{*}(t)=\left(0,0, \sqrt{1+t^{2}} e^{-t \arctan t}\right)$ which must be orthogonal to $\operatorname{Im} L_{\lambda}$, for any $\lambda \in S^{1}$.

Let us consider the perturbation $g(\lambda, t, u)=\left(0,0, u_{1}^{2}+u_{2}^{2}+u_{3}^{2}\right)$. By the above discussion, for any function $u \in H^{1}\left(\mathrm{R} ; \mathrm{R}^{3}\right)$ verifying $L_{\lambda} u-g(\lambda, t, u)=0$, we have $\int\left\langle u^{*}(t), g(\lambda, t, u(t))>d t=0\right.$. Hence $u(t)$ must vanish and no bifurcation arises. Notice that in this example we have Ind $L_{\lambda}=-1$, but it is clear how to construct examples of any index.

\section{REFERENCES}

1. Abbondandolo A., Majer P., Ordinary differential operators and Fredholm pairs, Math. Z. 243 (2003), 525-562. MR1970015 (2004d:58014)

2. Atiyah M. F., K-Theory, Benjamin, New York, 1967. MR0224083 (36:7130)

3. Berry M. V. , Geometric amplitude factors in adiabatic quantum transitions, Proc. Roy. Soc. London Ser. A 430 (1990), 405-411. MR1068305 (91k:81045)

4. Coppel W. A., Dichotomies in stability theory, Lect. Notes in Math. 629, Springer Verlag, Berlin 1978. MR0481196 (58:1332)

5. Crandall M. G., Rabinowitz P. H., Bifurcation from simple eigenvalues, J. Functional Analysis 8(1971), 321-340. MR0288640 (44:5836)

6. Fitzpatrick P. M., Pejsachowicz, J., Nonorientability of the index bundle and severalparameter bifurcation. J. of Functional Anal. 98 (1991), 42-58. MR1111193 (92g:58019)

7. Fitzpatrick P. M., Pejsachowicz J., Parity and generalized multiplicity, Trans. Amer. Math. Soc. 326 (1991), 281-305. MR1030507 (91j:58038)

8. Morris J. R., Nonlinear ordinary and partial differential equations on unbounded domains, PhD thesis, University of Pittsburgh, 2005.

9. Mrozek M., Reineck J. F., Srzednicki R., The Conley index over the circle, J. Dynam. Differential Equations 12 (2000), 385-409. MR1790660 (2002m:37022)

10. Palmer K. J., Exponential dichotomies and transversal homoclinic points, J. Diff. Eq. 104 (1988), 149-156. MR958058 (89k:34052)

11. Pejsachowicz J., Rabier P. J., Degree theory for $C^{1}$-Fredholm mappings of index 0, Journal d'Analyse Mathématique 76 (1998), 289-319. MR1676979 (2000a:58026)

12. Sacker R. J., The splitting index for linear differential systems, J. Diff. Eq. 33 (1979), no. 3, 368-405. MR543706 (81f:34020)

13. Secchi S., Stuart, C. A., Global bifurcation of homoclinic solutions of Hamiltonian systems, Discrete Contin. Dyn. Syst. 9 (2003), 1493-1518. MR2017678 (2005b:37138)

Dipartimento di Matematica, Politecnico di Torino, Corso Duca degli Abruzzi 24, 10129 TORINo, ITALY

E-mail address: jacobo.pejsachowicz@polito.it 